\title{
Comparative Analysis of Chemical, Fungal and Enzymatic Degradation of MCP
}

\author{
Jain $\mathrm{R}^{*}$ and Garg V \\ Department of Bioscience and Biotechnology, Banasthali University, Rajasthan, India
}

*Corresponding author: Jain R, Department of Bioscience and Biotechnology, Banasthali University, Rajasthan, Tel: 9410699471, E-mail: rachna11587@gmail.com

Citation: Jain R, Garg V (2014) Comparative Analysis of Chemical, Fungal and Enzymatic Degradation of MCP. SAJ Biotechnol 1: 101. doi: 10.18875/2375-6713.1.101

Article history: Received: 20 April 2014, Accepted: 15 July 2014, Published: 18 July 2014

\begin{abstract}
In general three different methods are used for the cleanup of pesticides. These include chemical, microbial and enzymatic methods. Therefore, the present study is an endeavor to compare the efficiency of all the three methods for the study of degradation of monocrotophos (MCP) $\left(150 \mu \mathrm{gml}^{-1}\right)$. Chemical method involved the use of $0.1 \mathrm{~N} \mathrm{NaOH}$, whereas for the biological as well as enzymatic method, molecularly characterized fungal strain Aspergillus niger JQ660373 was used. \%age of MCP degradation was assessed in the form of residual MCP concentration and hence degradation kinetics was premeditated. The results were confirmed by using analytical tools, High Performance Thin Layer Chromatography (HPTLC) and Fourier Transfom Infrared (FTIR). Results of the study demonstrated that degradation of MCP followed first order kinetics with the calculated half-life of 0.79 days in enzymatic method as compared to 3.16 and 4.21 days for fungal and chemical method. HPTLC chromatograms clearly indicate significant decrease in the standard MCP peak (rf 0.19-0.21) with increasing incubation duration. Molecular insight of MCP degradation as studied, suggests hydrolytic cleavage of MCP resulting in the formation of inorganic phosphates $\left(-\mathrm{PO}_{4}\right)$ and amines. The overall efficiency of degradation was found to be Enzymatic $>$ Fungal $>$ Chemical.
\end{abstract}

Keywords: Chemical; Organophosphorus; Pesticide; Degradation; Enzymes

\section{Introduction}

The term "pesticide" embraces an enormous diversity of products that are used in a number of different activities and are intended for preventing, destroying, repelling or mitigating pests. Among newly developed pesticides, organophosphorus pesticides (OPPs) are most commonly used as they degrade faster in the environment. MCP (Dimethyl (E)-1-methyl-2 methyl carbamoyl vinyl phosphate) is an organophosphate with broad-spectrum contact and systemic activity against spur throated locust, sorghum midge, western flower thrips, aphids, the green vegetable bug, mites, the stem borer, potato tuber moth which are common pests of sorghum, sunflowers, tomatoes, cotton, potato, soybean and tobacco [1,2].

Despite increasing crop productivity MCP is highly toxic to human beings. Mechanism of toxicity of MCP involves irreversible acetyl cholinesterase inhibition [3].

Owing to its highly toxic nature, degradation of MCP from the environment is very essential. Degradation of MCP occurs by biotic as well as abiotic factors of the natural environment. These factors influence the degradation of MCP and act in tandem and complement one another in the microenvironment [4].

Traditional cleanup methods of pesticides include the use of chemicals. These chemicals pose hazards on the soil fertility and soil microbial flaura and fauna. Therefore, these methods were discarded and biological methods emerged as a new tool for the degradation of pesticides.

Different microbial species such as algae, Chlorella vulgaris Beijernik, Scenedesmus bijugates, Synechococcus elongates, Nostac linckia and Phormedium tanue [5], several bacterial strains, Azospirillum lipoferum and Bacillus sp.[6], Arthrobacter atrocyaneus MCM B-425 and Bacillus megaterium MCM B-423 [2], Arthrobacter atrocyaneus, Bacillus megaterium, and Pseudomonas mendocina [7], Pseudomonas aeruginosa F10B and Clavibacter michiganense subsp. insidiosum SBL 11 [8], Paracoccus sp. M-1[9], Pseudomonas 
and Flavobacterium [10] are well known for their MCP degradation potential.

The main disadvantage of the use of bacteria for the degradation of pesticides is their short life span and low tolerance capacity to different environmental stresses. Hence, new technique of using fungi as a tool for the degradation pathway came into existence.

Fungi generally biotransform pesticides and other xenobiotics by introducing minor structural changes to the molecule and rendering it nontoxic. This bio-transformed pesticide is released into the soil, where it is susceptible to further degradation by bacteria [11].

Different fungal strains such as Aspergillus and Penicillium sp., [12], Penicillium corrylophylum [13], Aspergillus oryzae [14], Aspergillus sp. [15], Aspergillus niger JQ660373 [16], Aspergillus flavus, Fusarium pallidoroseum and Macrophomina sp. [17] are also very well known for their MCP degradation efficiency. Now a day's extracellular protein plays major role in the biodegradation of pesticides [18-22].

Different enzymes such as phosphatase and esterase [23,24], phosphotriesterase [8], carboxylesterase [25] and organophosphate hydrolase $(\mathrm{OPH})[26]$ have been reported to degrade MCP.

Therefore the present study is an endeavor to compare the MCP degradation rate by three distinct methods, chemical (0.1N $\mathrm{NaOH}$ ), microbial and enzymatic by using Aspergillus niger JQ660374 as a model microorganism.

\section{Materials and Method}

MCP of analytical grade (99.5\% purity) was purchased from sigma and stock solution ( $1 \mathrm{mgml}^{-1}$ in ethanol) was prepared. All the other chemicals used in this study were of analytical grade and were obtained from Himedia and Rankem, India.

Modified Czapekdox medium (CZM) was used as growth medium which contained sucrose, 30 g; NaNO, 2 g; KCl, 0.5 g; Mg$\mathrm{SO}_{4} .7 \mathrm{H}_{2} \mathrm{O}, 0.5 \mathrm{~g}$; glucose, $10 \mathrm{~g} ; \mathrm{FeCl}_{3}, 10 \mathrm{mg} ; \mathrm{BaCl}_{2}, 0.2 \mathrm{~g} ; \mathrm{CaCl}_{2}, 0.05 \mathrm{~g}$ per litre and was supplemented with $\mathrm{MCP}\left(150 \mu \mathrm{gml}{ }^{-1}\right)$ as a sole source of phosphorus.

Pure culture of Aspergillus niger was isolated from soil by serial dilution [16]. Hydrolases were purified from the fungal isolate by the method of [26].

\section{Experimental setup}

Chemical Method: $125 \mathrm{ml}$ of CZM medium was inoculated with $1 \mathrm{ml}$ of $0.1 \mathrm{~N} \mathrm{NaOH}$.

Microbial Method: Aspergillus niger JQ660373 was suspended in $1 \mathrm{ml}, 0.85 \%$ saline to make a cell suspension of $1 \times 10^{8}$ cells per $\mathrm{ml}$ and this suspension was inoculated in $125 \mathrm{ml}$ of CZM medium [16].

Enzymatic Method: Aspergillus niger JQ660373 at a concentration of $1 \times 10^{8}$ cells per ml was suspended in CZM medium and incubated for 10 days in an orbital shaking incubator at $28 \pm 2{ }^{\circ} \mathrm{C}$. After the completion of incubation time the flasks were withdrawn and the filrate was checked for the presence of hydrolase enzyme by the assay procedure described by [26]. Extracellular enzyme was purified by $\mathrm{AmSO}_{4}$ precipitation, dialysis and G- 100 Chromatography. Partially purified enzyme (G-100) was further purified by ion exchange chromatography. The concentration of the purified enzyme was optimized by estimating the enzyme activity at different protein concentrations i.e. 50, 100, 150, 200, 250 and $300 \mu \mathrm{gml}^{-1} .1 \mathrm{ml}$ (optimum concentration) of the purified enzyme fraction was inoculated in $125 \mathrm{ml}$ of CZM medium [26].

Effective antibiotics, streptomycin and penicillin $\left(30 \mathrm{mgL}^{-1}\right)$, were added in the growth medium to avoid bacterial contamination. Control sample containing CZM media $+\mathrm{MCP}\left(150 \mathrm{mgL}^{-1}\right)$ were prepared simultaneously. All the flasks are incubated at $28 \pm 2{ }^{\circ} \mathrm{C}$ for 15 days in an orbital shaking incubator at $90 \mathrm{rpm}$ under aerated culture conditions.

At regular time interval of 2 days, the flasks were withdrawn and the samples were subjected to extraction and cleanup by equal amount of ethyl acetate and florosil column. The residual MCP was quantified by spectrophotometer at $254 \mathrm{~nm}$.

$\%$ of MCP degradation was calculated using the formula

\section{$\%$ of MCP Degradation $=\underline{\text { Concentration of MCP in control- } \text { Concentration of MCP in sample }} \times 100$ Concentration of MCP in control}

Kinetics of degradation was studied by plotting logarithmic residual MCP concentration as a function of time [16].

The results were further confirmed by HPTLC and FTIR.

Analytical Method: High Performance Thin Layer Chromatography (HPTLC) (CAMAG Linomat 5, Switzerland) was used for analysis of samples. The aqueous samples from MCP degradation flasks were extracted with ethyl acetate. The samples (20-25 $\mathrm{ml}$ ) were inoculated on silica gel (60 F 254) TLC plates (E. Merck, India) using an applicator system. A twin trough glass chamber 
$(20 \times 20 \mathrm{~cm})$ containing Dichloromethane: Methanol (9:1) as the mobile phase was used for development of the chromatogram. Spots were detected using a CAMAG TLC scanner-3 at the wavelength of $254 \mathrm{~nm}$ using a deuterium lamp.

Infrared spectra of the parent compound (MCP) and sample after fungal degradation were recorded at room temperature $\left(25^{\circ} \mathrm{C}\right)$ in the frequency range of 4000-400 $\mathrm{cm}^{-1}$ with a Fourier transform infrared (FTIR) spectrophotometer ( 8400 Shimadzu, Japan, with Hyper IR-1.7 software for Windows) with a helium neon laser lamp as a source of infrared radiation. Aqueous samples (96 h of incubation) from MCP degradation flasks were extracted with ethyl acetate and solvent was evaporated using a rotary vacuum evaporator (Rotavapor R.214, Bu"chi, Switzerland). The contents were re-dissolved in acetone. A drop of this sample in acetone was placed in between two sodium chloride discs, after cleaning with ethyl acetate. The background spectrum for acetone was corrected from the sample spectrum.

All the experiments were carried out in triplicates. The statistical analysis was done by using Statistical Package for the Sciences System 17 (SPSS 17). The variables were subjected to Student t-test and One Way ANOVA.

\section{Results}

Aspergillus niger was grown in CZM medium containing $0.5 \mathrm{gmL}^{-1} \mathrm{KH}_{2} \mathrm{PO}_{4}$ for the production of extracellular enzyme secreted by the isolates. After 10 days of incubation period the medium was checked for the presence of extracellular enzyme. Intracellular hydrolase activity was also checked. It was found that Aspergillus niger possessed potent extracellular hydrolase activity (10.34 \pm $0.2 \mathrm{U}$ and Sp. activity $\left.3.42 \mathrm{Umg}^{-1}\right)$ in comparison to intracellular activity $\left(2.31 \pm 0.01 \mathrm{U}\right.$ and Sp. Activity $\left.1.12 \mathrm{Umg}^{-1}\right)$. Therefore the secreted fraction was collected and was partially purified by ammonium sulphate precipitation and gel filtration on Sephadex G100 and ion exchange chromatography. The data for the purification of the extracellular OP hydrolase was summarized in table 1.

\begin{tabular}{|c|c|c|c|c|c|c|}
\hline & & $\begin{array}{l}\text { Total activity } \\
\text { (U) }\end{array}$ & $\begin{array}{c}\text { Total protein } \\
(\mathrm{mg})\end{array}$ & $\begin{array}{c}\text { Sp. Activity } \\
\left(\mathrm{U} \mathrm{mg}^{-1}\right)\end{array}$ & Purification fold & Yield \% \\
\hline Crude & & $812.63 \pm 2.81$ & $351.33 \pm 5.97$ & $2.31 \pm .034$ & 1 & 100 \\
\hline \multirow{2}{*}{$\mathrm{AmSO}_{4} \mathrm{ppt}$} & Intracellular & $152.13 \pm 3.36$ & $112.21 \pm 3.79$ & $1.35 \pm 0.01$ & $0.58 \pm 0.04$ & $18.72 \pm 0.2$ \\
\hline & Extracellular & $553.73 \pm 3.52$ & $221.9 \pm 8.45$ & $2.49 \pm .11$ & $1.07 \pm .03$ & $68.14 \pm .67$ \\
\hline \multirow{2}{*}{ G-100 } & OPH 33 & $320.12 \pm 4.46$ & $5.26 \pm .35$ & $60.85 \pm 3.46$ & $26.34 \pm 2.34$ & $39.39 \pm 1.16$ \\
\hline & OPH 67 & $450.02 \pm 2.64$ & $5.26 \pm .35$ & $85.69 \pm 6.29$ & $37.04 \pm 3.07$ & $55.37 \pm .16$ \\
\hline \multirow{2}{*}{ DEAE CL6B } & ОРН 33 & $297.14 \pm 5.52$ & $2.15 \pm 0.01$ & $138.2 \pm 2.42$ & $59.82 \pm 1.14$ & $40.32 \pm 1.34$ \\
\hline & OPH 67 & $425.11 \pm 4.46$ & $2.26 \pm 0.04$ & $188.1 \pm 3.36$ & $81.42 \pm 2.34$ & $52.31 \pm 1.13$ \\
\hline
\end{tabular}

Table 1: Purification profile of extracellular fungal hydrolases from Aspergillus niger.

It shows that the purified enzyme showed a purification fold of $37.04 \pm 3.07$ with a percent yield of $55.37 \pm 0.16$. The enzyme activity was found to be $450.02 \pm 2.64 \mathrm{U}$ with the protein content of $5.26 \pm 0.35 \mu \mathrm{gml}^{-1}$. Hence the calculated specific activity of purified enzyme was $85.69 \pm 6.29 \mathrm{Umg}^{-1}$. Purified OP hydrolase composed of two different subunits. The molecular mass of each of the subunits as estimated by SDS PAGE analysis as shown in Figure 1 depicts that the enzyme was composed of two different subunits. These were found to be of approximately $33 \mathrm{Kda}$ and $67 \mathrm{Kda}$ molecular weight.

(1)

(2)

(3)

(4)

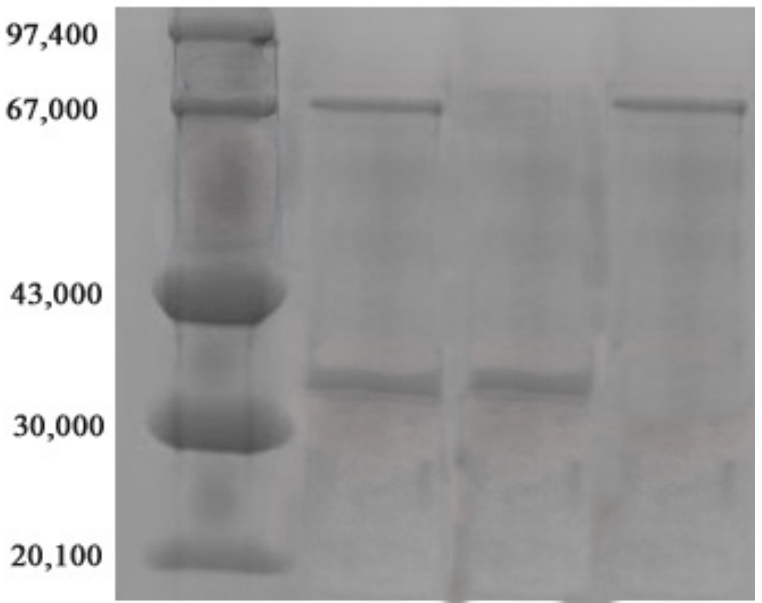

Figure 1: SDS-PAGE of the partially purified extracellular enzyme from five isolated strains. Lane 1, marker proteins (from top to bottom) phosphorylase $\mathrm{b}$ ( $\mathrm{Mr}$, $97,400)$, bovine serum albumin $(M r, 67,000)$, ovalbumin $(M r, 43,000)$, carbonic anhydrase $(M r, 30,000)$ and Soyabean Trypsin Inhibitor $(M r, 20,100)$, respectively; lane 2, purified enzyme G-100, lane 3, low molecular weight protein DEAE CL6B, lane 4, high molecular weight protein DEAE CL6B. The gel was stained for protein with Coomassie brilliant blue R-250 and destained in methanol-acetic acid-water (7:2:1). 
As depicted in the previous section hydrolase enzyme composed of two different subunits of different molecular weights i.e 33 and $67 \mathrm{Kda}$ respectively. Therefore both these purified subunits fractions (individual as well as combined) were tested for their optimum concentration for the efficient degradation of MCP. The enzyme activity of all the three different protein fractions followed an L-shaped pattern. It is evident from figure 2 that $150 \mu \mathrm{gml}^{-1}$ is the optimum concentration among all the tested enzyme concentrations. Therefore, this selected concentration was used for further degradation study. Further it was also observed that combined fraction of both subunits showed high efficiency towards MCP degradation and hence showed high enzyme activity. Therefore, this fraction was further selected for the degradation of MCP.

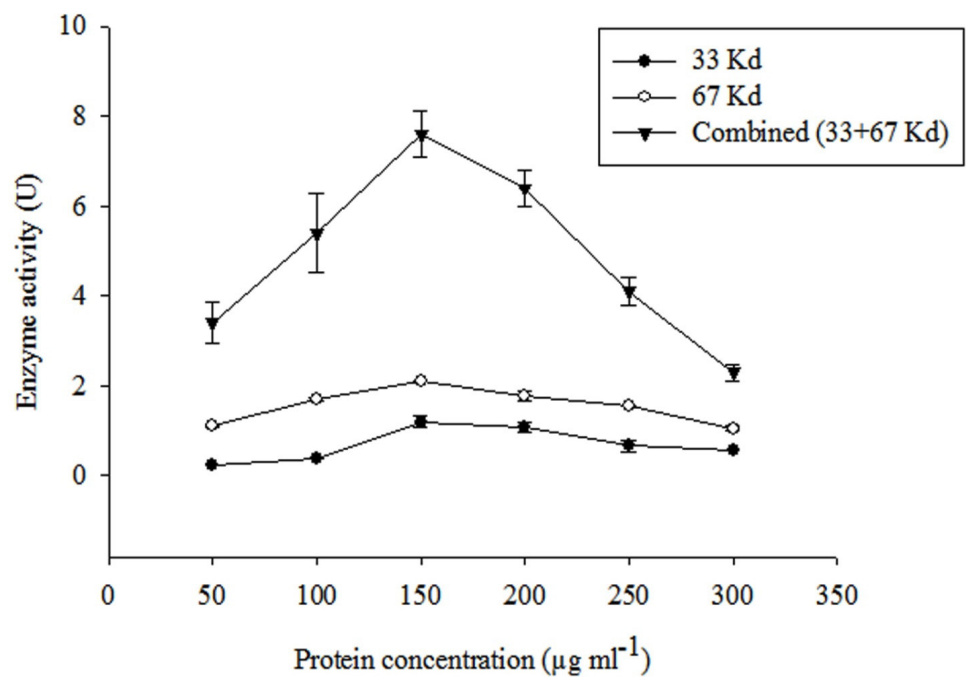

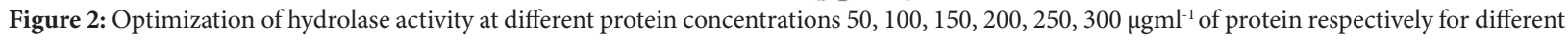
subunits of purified M1 viz. 33, 67 and both combined. Error bars indicates standard deviation.

Degradation of MCP in phosphorus free liquid medium was studied by inoculating $1 \mathrm{ml}$ each of $0.1 \mathrm{~N} \mathrm{NaOH}$, spore suspension and combined purified enzyme fraction. Residual MCP was extracted thrice with equal amount of ethyl acetate at an interval of 2 days. It is clearly evident from figure 3 that residual MCP concentration decreases exponentially with increasing incubation period. Maximum decrease was found in the enzymatic method followed by microbial and chemical. Residual MCP concentration was found to be $40.77 \pm 1.34,2.45 \pm 0.45$ and $0.12 \pm 0.001 \mu \mathrm{g} \mathrm{ml}^{-1}$ for chemical, fungal and enzymatic methods respectively after 10 days of incubation.

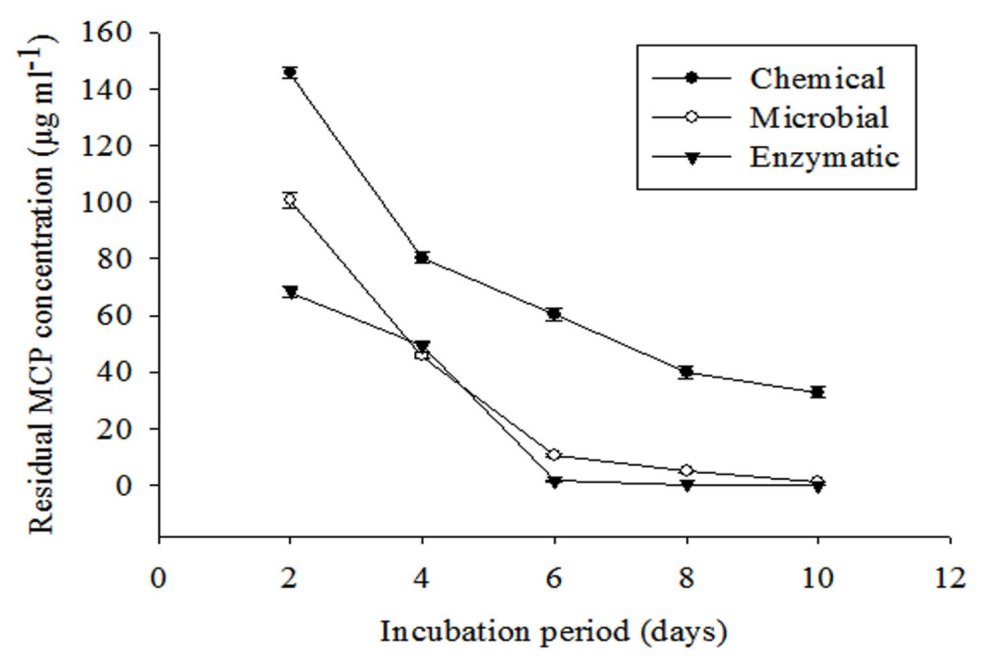

Figure 3: Residual MCP concentration $\left(\mu \mathrm{gml}^{-1}\right)$ by chemical, fungal and enzymatic method at various incubation periods in phosphorus free liquid culture medium. Error bars indicates standard deviation.

The results of spectrophotometric study were further confirmed by HPTLC. Figure 4 clearly indicate the visible significant decrease in the standard MCP peak with increasing incubation duration. Standard MCP peak was found at rf 0.19-0.21. No such peak was found after 10 days of incubation for enzymatic mode of degradation whereas, for chemical and fungal method short peaks were observed. This clearly indicates complete detoxification of MCP by extracellular fungal hydrolases from Aspergillus niger JQ660373. In addition to the standard MCP peak some other peaks were also observed which were characteristic of the by-products of MCP degradation. However these were not identified as much emphasis was given on parent compound degradation. 
FTIR spectrum of MCP degradation as shown in figure 5 clearly indicates hydrolytic cleavage of MCP with the formation of inorganic phosphates $\left(-\mathrm{PO}_{4}\right)$. Peaks at $3406 \mathrm{~cm}^{-1}$ and $3263 \mathrm{~cm}^{-1}$ as visible in control samples were characteristic for vinyl bonds. These peaks were completely reduced in the spectrum after degradation. Whereas, a new peak at $1398.71,1385.11$ and $1367.93 \mathrm{~cm}^{-1}$ was observed characteristic of inorganic phosphates. Some other peaks at 1063.63, 1073.54 and $1067.24 \mathrm{~cm}^{-1}$ were also observed which were characteristic for aliphatic amines. Some new peaks at a range of $1497.64-1543.33 \mathrm{~cm}^{-1}$ characteristic for $-\mathrm{NH}$ or $-\mathrm{NO}$ were also pragmatic. It is also depicted from the figure that the number of peaks increased with enzymatic method as compared to fungal and chemical method as employed in the present study. It also signifies the efficiency of enzymatic method towards degradation of MCP.

Degradation kinetics of MCP followed straight line equation (Figure 6). Hence the rate constant and half life was calculated by using first order kinetics. The kinetic rate constants were observed to be $0.0069,0.0092$ and 0.037 day- 1 and hence the calculated half life of MCP was $0.79,3.16$ and 4.21 days respectively for chemical, fungal and enzymatic methods respectively. The half life of MCP was significantly high (infinite) in control sample.
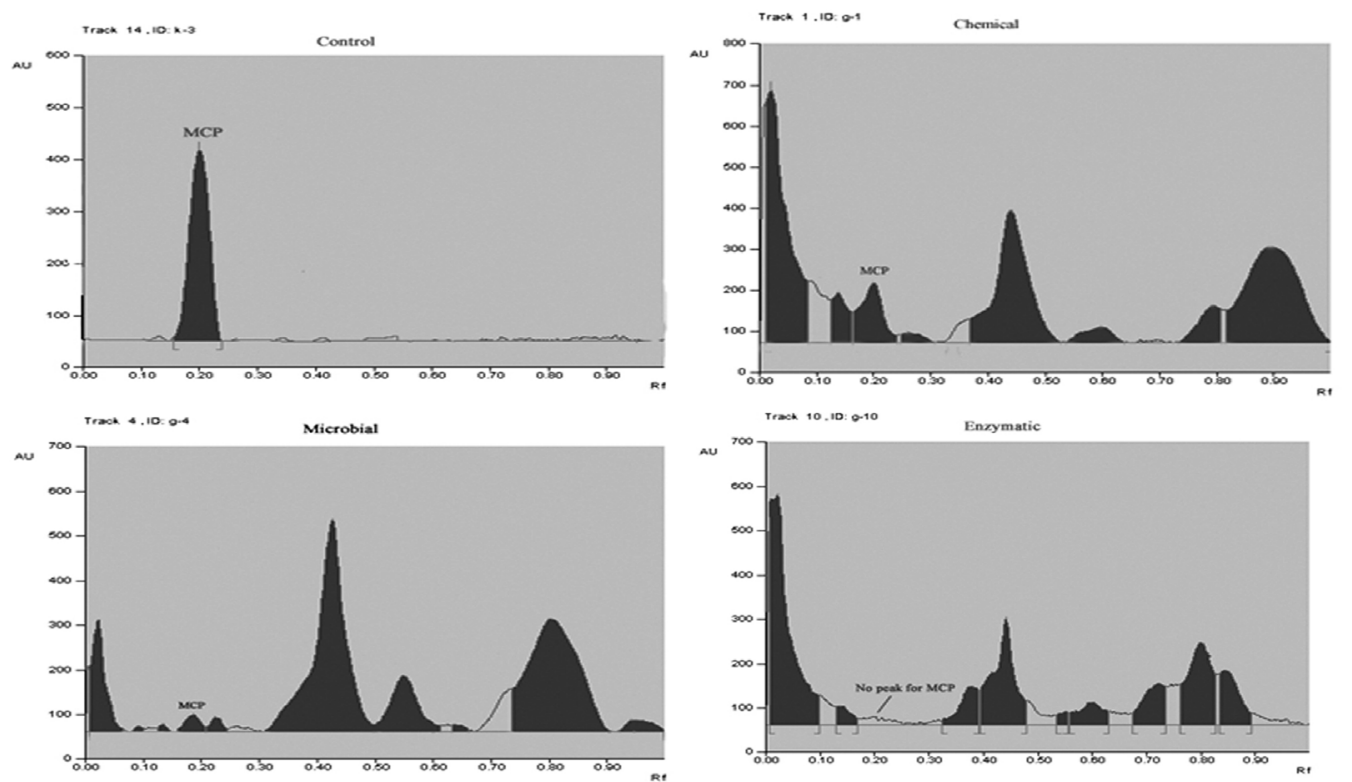

Figure 4: HPTLC chromatograms of residual MCP concentration in CZM medium by (a) Control, (b) Chemical, (c) Microbial and (d) Enzymatic after10 days of incubation.
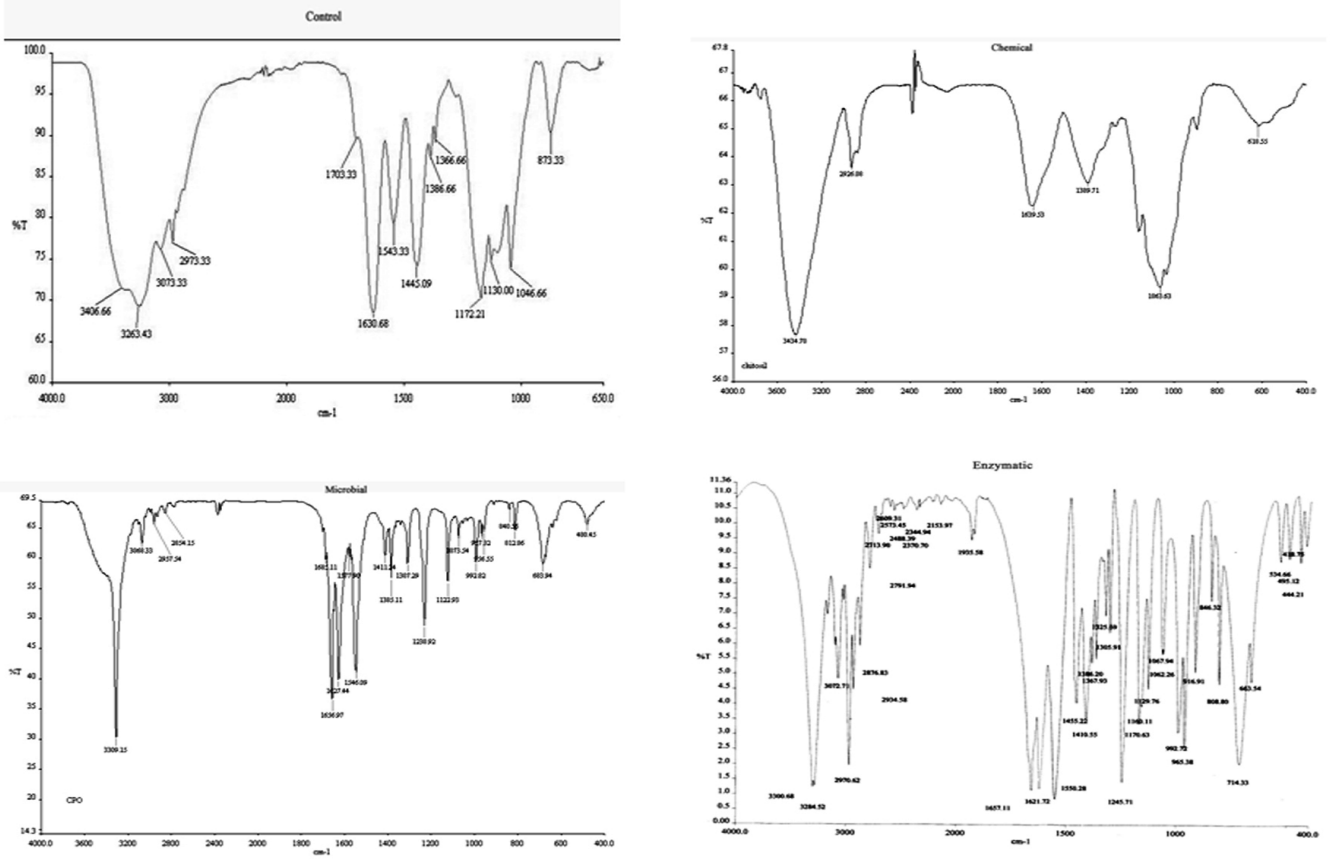

Figure 5: FTIR spectrum of MCP degradation by (a) Control, (b) Chemical, (c) Microbial and (d) Enzymatic methods in CZM medium after 10 days of incubation. 

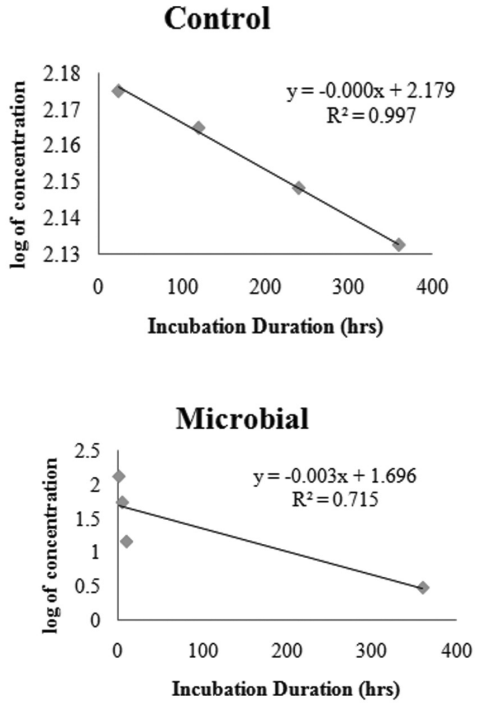



Enzymatic

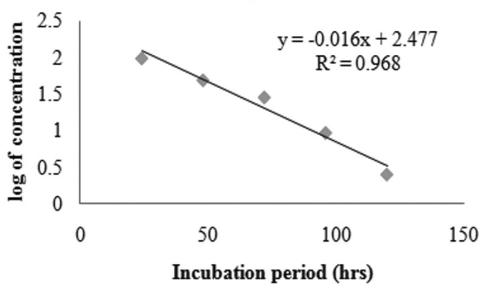

Figure 6: Degradation kinetics of MCP by (a) Control, (b) Chemical, (c) Microbial and (d) Enzymatic methods in CZM medium at different incubation periods. Straight line equation shows that degradation of MCP follows first order kinetics.

\section{Discussion}

The present study is a comparative endeavor to find out the most efficient method for the degradation of MCP among the three commonly used methods viz. chemical, fungal and enzymatic. Molecularly identified fungal strain Aspergillus niger JQ660373 was used in the present study [16]. In the chemical method $0.1 \mathrm{~N} \mathrm{NaOH}$ was used, as alkaline hydrolysis is a common method for the degradation of MCP [27]. $1 \mathrm{ml}$ each of $0.1 \mathrm{~N} \mathrm{NaOH}$, fungal spore suspension and purified enzyme fraction was inoculated in $125 \mathrm{ml}$ of phosphorus free CZM medium for 10 days. It was observed that MCP was degraded exponentially and rapidly by enzymatic and fungal methods whereas, for chemical method the rate of degradation was low. After 5 days there was observed a lag period in case of fungal method and therefore comparative rate of degradation of MCP was found higher for enzymatic than for fungal method.

This clearly indicates that pure enzyme fraction could be easily used at contaminated sites for the removal of MCP. During chemical degradation MCP gets converted into N-methylacetoacetamide, which can be either directly measured or indirectly by the disappearance of MCP from the solution. Metabolic reactions taking place during the metabolism of MCP by microbial cultures are $\mathrm{N}$-demethylation, O-demethylation, hydroxylation of $\mathrm{N}$-methyl groups and cleavage of the phosphate-crotanamide linkage, $[23,28,29]$, with the formation of O-desmethyl MCP monomethyl phosphate, dimethyl phosphate, N-methyl acetoacetamide and $\mathrm{N}$-methylbutyramide. Although the basic mechanism of MCP degradation by enzyme fraction is not exactly known but it can be presumed that firstly MCP is broken down into secondary metabolite and then it is completely mineralized.

Fungal strain was tested for its intra as well as extracellular enzyme content. It was found that the strain possessed high extracellular enzyme activity. The high enzyme activity fraction was then purified by G-100 chromatography and SDS- PAGE analysis depicted that the enzyme was composed of two subunits of different molecular weight [26]. Both enzyme fractions were then separated by ion exchange chromatography.

Both fractions were individually as well as combined tested for their MCP degradation efficiency. It was observed that the combined fraction degrade MCP very effectively. There may be some synergistic association between these two fractions for the degradation of MCP.

To the best of our knowledge, it is the first study comparing the degradation rate of MCP by chemical, fungal and enzymatic methods. However, one such study was reported by [30], in which he compared the degradation rate of crude cell extract of mixed microbial culture growing on parathion with that of $0.1 \mathrm{~N} \mathrm{NaOH}$. He had reported that rate of enzymatic hydrolysis of parathion was 2,450 times faster than that of the chemical method. He had also compared the activity of this crude enzyme preparation on other seven organophosphate pesticides viz. trizaphos, paraoxon, EPN, diazinon, methyl parathion, dursban, fenitrothion, cyanophos, azinophos-M, ethion, chlromephos and fenthion and reported that the rate of enzymatic hydrolysis was $1005,525,11$, $43,143,122,40,205$ and 73 times higher than that of the rate of chemical hydrolysis.

Although there are a number of individual studies on the degradation of MCP by different fungal isolates and enzymes but studies on chemical hydrolysis of MCP are scarce. [27] had studied alkaline hydrolysis of MCP in blood samples. 
73.33, 98.36 and $99.25 \%$ of MCP was degraded within 10 days of incubation by chemical, fungal and enzymatic method. [17] had reported 91, 75 and 79\% of MCP degradation by Aspergillus flavus, Fusarium pallidoroseum and Macrophomina sp. within 15 days of incubation. [14], 2009 had reported the ability of Aspergillus oryzae to completely mineralize MCP (1000 mgL-1) in M1 medium within 168 hours of incubation. Similarly [12] had also reported 75\% and 50\% degradation of MCP (200 $\left.\mathrm{mgL}^{-1}\right)$ within 4 days by Aspergillus and Penicillium sp. respectively.

On the other hand [26] had also reported efficient and rapid degradation of MCP by five distinct extracellular hydrolases isolated from five different fungal isolates.

The results were further analyzed by HPTLC which clearly demonstrate significant reduction in the standard peak of MCP (rf 0.19-0.21). Some new peaks were also seen in addition to the standard peak which might be due to the metabolic products of MCP degradation. However, these were not identified as the emphasis was given on the disappearance of MCP by fungal isolates. Molecular insight of MCP degradation clearly indicated the cleavage of vinyl bond with the formation of new peaks characteristic of $-\mathrm{PO}_{4}$ in the FTIR spectrum. The results were concurrent with the study of $[14,17,26]$ which also correlated degradation of MCP by reduction of its peak as compared to that in the standard chromatogram by hydrolytic cleavage.

Similar to the earlier reports of [14,16,26,30,31] degradation of MCP followed first order kinetics with a half life of 0.79 days in enzymatic method as compared to 3.16 and 4.21 days for fungal and chemical method. This clearly demonstrates the efficiency of extracellular enzymes for MCP degradation. Concurrent with the studies of $[17,26]$ the half life of control sample was found to be infinite and therefore clearly ruled out the possibility of auto degradation.

Concurrent to our study [17] had reported the half-life of MCP to be 4.21, 12.64 and 6.32 days for Aspergillus flavus, Fusarium pallidoroseum and Macrophomina sp. respectively. Simultaneously, [16] had reported the half-life of MCP as $75.87 \mathrm{~h}$ by Aspergillus niger. The calculated half life of MCP was found to be $0.79,2.11,0.6,1.8$, and 2.11 days respectively for five distinct fungal hydrolases by [26].

Hence the study concludes that enzymes proved themselves to be the most efficient tool for the degradation of MCP in liquid culture medium. The overall order of degradation effeciency was found to be Enzymatic $>$ Fungal $>$ Chemical.

\section{Acknowledgment}

This work was financially supported by the Department of Bioscience and Biotechnology, Banasthali University, Rajasthan, India, that is gratefully acknowledged.

\section{References}

1. Vig K, Singh DK, Agarwal HC, Dhawan AK, Dureja P (2001) Insecticide residues in cotton crop soil. J Environ Sci Health B 36: 421-34.

2. Bhadbhade BJ, Dhakephalkar PK, Sarnaik SS, Kanekar PP (2002) Plasmid-associated biodegradation of an organophosphorus pesticide, MCP, by Pseudomonas mendocina. Biotechnol Lett 24: 647-50.

3. Jokanovic M (2001) Toxicology (In Serbian), University textbook, Elit-Medica, Belgrade.

4. Singh BK, Walker A, Morgan JAW, Wright DJ (2003) Effects of soil pH on the biodegradation of chlorpyrifos and isolation of a chlorpyrifos-degrading bacterium. App Environ Microbiol 69: 5198-206.

5. Megharaj M, Venkateswarlu K, Rao AS (1987) Metabolism of MCP and quinalphos by algae isolated from soil. Bull Environ Contam Toxicol 39: 251-6.

6. Rangaswamy V, Venkateswarlu K (1992) Activities of amylase and invertase as influenced by the application of MCP, quinalphos, cypermethrin and fenvalerate to groundnut soil. Chem 25: 525-530.

7. Bhadbhade BJ, Sarnaik SS, Kanekar PP (2002) Bioremediation of an industrial effluent containing monocrotophos. Curr Microbiol 45: 346-9.

8. Subhas, Singh DK (2003) Utilization of monocrotophos as phosphorus source by Pseudomonas aeruginosa F10B and Clavibacter michiganense subsp. insidiosum SBL 11. Can J Microbiol 49: 101-9.

9. Jia Kai-zhi, Cui Zhong-li, He Jian, Guo Peng, Li Shun-pen (2006) Isolation and characterization of a denitrifying MCP degrading Paracoccus sp.M-1. FEMS Microbiol Lett 263: 155-62.

10. Nazarian A (2007) Utilization of Dimethoate by Wild Type Pseudomonas Putida from polluted Sites in Iran. Pak J Biol Sci 10: 664-7.

11. Gianfreda L, Rao MA (2004) Potential of extra cellular enzymes in remediation: A review. Enzyme Microbial Technol 35: 339-54.

12. Zidan ZH, Ramadan EM (1976) Degradation of some organophosphorus insecticides. Egypt J Microbiol 11: 93-9.

13. Rabie GH (1995) Biodegradation of the organophosphorus insecticide MCP by Penicillium corrylophylum. Zagazig J Pharmacological Sci 4: 14-9.

14. Bhalerao S Tejomyee, Puranik R Pravin (2009) Microbial degradation of monocrotophos by Aspergillus oryzae. Int Biodeterior Biodeg 63: 503-8.

15. Anitha S, Das MSS (2011) Mycoremediation of MCP. Int J Pharma Bio Sci 2: 337-42.

16. Jain R, Garg V, Singh KP, Gupta S (2012) Isolation and characterization of monocrotophos degrading activity of soil fungal isolate Aspergillus Niger MCP1 (ITCC7782.10). Int J Environ Sci 3: 841-50.

17. Jain R, Garg V, Yadav D (2014) In vitro Comparative Analysis of MCP Degrading Potential of Aspergillus flavus, Fusarium pallidoroseum and Macrophomina sp. Biodegradation 25: 437-46.

18. Glenn JK, Gold MH (1985) Purification and characterization of an extracellular Mn (II) dependant peroxidase from the lignin degrading basidiomycete Phanerochaete chrysosporium. Ach of Biochem 242: 329-41. 
19. Tarafdar JC, Rao AV, Bala K (1988) Production of phosphatases by fungi isolated from desert soils. Folia Microbiol 33: 453-7.

20. Abd-Alla MH (1994) Use of organic phosphorus by Rhizobium leguminosarum biovar viceae phosphatase. Biol Fertile Soils 18: $216-8$.

21. Abd-Alla MH (1994) Phosphodiesterase and phosphotriesterase in Rhizobium and Bradyrhizobium strains and their roles in the degradation of organophosphorus pesticides. Lett App Microbiol 19: 240-3.

22. Rana BK, Johri BN, Thakur IS (1996) Formation and activities of xylan-hydrolyzing enzymes of Humicola grisea var thermoidea. World J Microbiol Biotechnol

12: $12-5$.

23. Bhadbhade BJ, Sarnaik SS, Kanekar PP (2002) Biomineralization of an organophosphorus pesticide, monocrotophos, by soil bacteria. J Appl Microbiol 93: 224-34.

24. Srinivas RR, Jayalakshmi SK, Sreeramulu K (2004) Hydrolysis of organophosphorus compounds by an esterase isozyme from insecticide resistant pest Helicoverpa armigera. Indian J Exp Biol 42: 214-6.

25. Qiao Ch.-L, Huang J, Li X, Shen BCh, Zhang JL (2003) Bioremediation of Organophosphate Pollutants by a Genetically-Engineered Enzyme. Bull Environ Contam Toxicol 70: 455-61.

26. Jain R, Garg V (2013) Enzymatic degradation of MCP by extracellular fungal OP hydrolases. Appl Biochem Biotechnol Appl Biochem Biotechnol 171: 147386.

27. Janghel EK, Rai JK, Rai MK, Gupta VK (2006) A New and Highly Sensitive Spectrophotometric Determination of MCP in Environmental, Agricultural and Biological Samples. J Chinese Chem Soc 53: 343-47.

28. Guth JA, Voss G (1970) Photodecomposition of Dichlorvos, Phosphamidon, Dicrotophod, Monocrotophos, Dioxacarb, C10015, and C17018 in Aqueous and Methanolic Solutions. Ciba Ltd, Switzerland.

29. Gundi AKB Vijay, Reddy BR (2006) Degradation of monocrotophos in soils. Chem 62: 396-403.

30. Munnecke DM (1976) Enzymatic hydrolysis of organophosphate insecticides, a possible pesticide disposal method. App Environ Microbiol 32 : 7-13.

31. Tariq MS Dawar M Abid, Shaukat SS (2005) Seed-borne mycoflora of soyabean. Int J Biol and Biotechnol 2: 711-3. 